\title{
Task coordination between and within sensory modalities: Effects on distraction
}

\author{
MURIELE BRAND-D'ABrescia \\ University College London, London, England \\ and Université de Provence, Aix en Provence, France \\ AND \\ NiLli Lavie \\ University College London, London, England
}

\begin{abstract}
Load theory predictions for the effects of task coordination between and within sensory modalities (vision and hearing or vision only) on the level of distraction were tested. Response competition effects in a visual flanker task when it was coordinated with an auditory discrimination task (between-modality conditions) or a visual discrimination task (within-modality conditions) were compared with single-task conditions. In the between-modality conditions, response competition effects were greater in the two- (vs. single-) task conditions irrespective of the level of discrimination task difficulty. In the within-modality conditions, response competition effects were greater in the two-task (vs. single-task) conditions only when these involved a more difficult visual discrimination task. The results provided support for the load theory prediction that executive control load leads to greater distractor interference while highlighting the effects of task modality.
\end{abstract}

It has long been thought that executive control functions associated with the frontal lobe (such as working memory) play a role in goal-directed control of attention (Baddeley, 1996; Desimone \& Duncan, 1995). However, behavioral experiments have often failed to show any specific effect of working memory load on performance in attention tasks (e.g., Logan, 1978; Woodman, Vogel, \& Luck, 2001; but see Han \& Kim, 2004, and Woodman \& Luck, 2004, for exceptions). By contrast with the previous failures, a recent series of studies examining the load theory of attention has provided direct evidence for a specific causal role of executive control in maintaining focused attention on task-relevant stimuli while preventing distraction by task-irrelevant stimuli (de Fockert, Rees, Frith, \& Lavie, 2001; Lavie \& de Fockert, 2005, 2006; Lavie, Hirst, de Fockert, \& Viding, 2004).

Load theory proposes two means of control of selective attention that serve to prevent the processing of irrelevant and potentially distracting stimuli. The first is a fairly passive means of control, whereby task-irrelevant stimuli are simply not perceived when task-relevant processing involves a sufficiently high perceptual load that exhausts perceptual capacity with the perception of task-relevant stimuli. The second is an active means of executive control, whereby the active maintenance of stimulus-processing priorities minimizes distraction by low-priority task-irrelevant stimuli even when these have been perceived (in a situation of low perceptual load that leads to a spillover of capacity and, thus, results in distractor perception). This hypothesis leads to the prediction that when executive control is loaded (e.g., with the need to actively maintain stimuli in an added working memory task or with the need to coordinate swift shifting from one task to another), this should result in greater interference effects by task-irrelevant distractors.

Support for the perceptual mechanism of control hypothesized in load theory has been found in many studies showing elimination of distractor processing in tasks with a high perceptual load (e.g., Lavie, 1995; see Lavie, 2005, for a review). Support for the role of executive control in attention stipulated in load theory has also been found in recent experiments (Lavie, 2000; Lavie \& de Fockert, 2005, 2006; Lavie et al., 2004) that have shown that distractor interference effects (measured with response competition effects produced by irrelevant distractors in the flanker task [Eriksen \& Eriksen, 1974] or with the attentional capture effects produced by an irrelevant color singleton during a shape-based visual search task) and related visual cortex activity (see de Fockert et al., 2001) were increased when executive control was loaded with an additional working memory task (as compared with low working memory load conditions or with a single-task performance of the selective attention task).

All these experiments involved a working memory task and a selective attention task, and the material presented

N. Lavie, n.lavie $@$ ucl.ac.uk 
in both the selective attention and the working memory tasks was visual. The conclusions are, therefore, restricted to effects of executive load on tasks involving explicit demands on working memory and sharing the same sensory (visual) modality.

Here, we sought to examine load theory claims about the role of executive control in determining the level of distraction further by varying executive control load with coordination of perceptual discrimination tasks involving either the same (visual) or different (visual and auditory) sensory modalities. Although the coordination of performance in two perceptual discrimination tasks does not explicitly load working memory, the demand placed on executive control by the need to coordinate two different tasks with different task rules should reduce its ability to provide goal-directed control of the processing in each task and, hence, should result in greater interference by goal-irrelevant distractors. Indeed, we have recently reported greater interference by irrelevant distractors both in the response competition paradigm and in the attentional capture paradigm in conditions of task switching, as compared with task repetition (Brand \& Lavie, 2005). But the material used in this study was purely visual.

In the present study, our aim was to test the effects of twotask coordination on distractibility in tasks involving different sensory modalities (vision and hearing) and to compare them with within-modality (vision-only) tasks. Executive control functions that serve to actively maintain stimulus-processing priorities and response-mapping rules are conceived as highlevel cognitive processes typically mediated by the frontal cortex well beyond sensory processes in modality-specific cortices (e.g., the occipital cortex for vision). Thus, the effects of executive control load on distraction should not be restricted to tasks involving the same sensory modality. We thus hypothesized that the mere demand placed on executive control by the need to coordinate two different tasks should reduce its ability to provide goal-directed control that prevents interference by goal-irrelevant distractors, irrespective of the sensory modality of each task.

To test this hypothesis, we assessed response competition effects from irrelevant distractors in a visual flanker task (similar to that used in the previous within-modality executive load experiments by Lavie and colleagues) as a function of executive load induced either by the need to coordinate two tasks within the same (visual) modality or across (visual and auditory) modalities. Load on executive control was manipulated in a way similar to that in Lavie et al. (2004, Experiments 4 and 5) by varying whether the flanker task was performed alone in the single-task conditions or combined with another task in succession on each trial in two-task conditions. As was mentioned earlier, in a change from Lavie et al. (2004), we did not use a working memory task in the two-task conditions, since maintenance in working memory may involve a modality different from the presentation modality (e.g., alphanumeric stimuli are likely to be maintained using a phonological code even when presented visually). In order to clearly identify the stimulus modality in the task that needs to be coordinated with the flanker task, we used, instead, either an auditory discrimination task (in the cross-modal two-task conditions) or a visual discrimination task (in the within-modality two-task conditions), in addition to the visual flanker task.

\section{EXPERIMENT 1}

In Experiment 1, we compared response competition effects produced by the distractor in a flanker task between a single-task condition and a between-modality two-task condition in which an auditory discrimination task had to be coordinated with the visual flanker task. The subjects listened to a stream of tones, detecting whether a target tone with a lower frequency than the other tones had a long or a short duration. As in Lavie et al. (2004, Experiments 4 and 5), the flanker task immediately followed. The hypothesis that active maintenance of the stimulus-processing priorities that minimizes distraction by irrelevant stimuli in one task would be loaded by the need to coordinate alternation between a task in another modality that had a different set of stimulus-processing rules led us to predict greater interference by the visual distractors in the flanker task immediately following the auditory task in the two-task condition, as compared with the single-task condition.

\section{Method}

\section{Subjects}

Eighteen students from the UCL subjects pool were paid at a rate of $£ 6 / \mathrm{h}$ for participation in the experiment. The age range of all the subjects in the experiments reported was 18-35 years old, and they all reported normal or corrected-to-normal vision and no auditory impairment.

\section{Stimuli}

The experiments were created and run on a PC using E-Prime ( 1.0 beta 5.0 version), sold by Psychology Software Tools. Auditory stimuli were created using the SoundEdit 16 software package, sold by Macromedia. Auditory stimuli were presented through Beyer open-cup headphones. The target and nontarget tones were presented at an intensity of $72 \mathrm{~dB}$ SPL. The nontarget tones were of $520-\mathrm{Hz}$ frequency, and the target tone's frequency was $440 \mathrm{~Hz}$. The target and nontarget tones were equally likely to be of either 100 or $150 \mathrm{msec}$ in duration. Each trial's sound sequence contained two long and two short nontarget sounds and one target (short or long), all presented in random order, with the constraint that the target was never presented in the first sequence position.

In the flanker task, letters were displayed in black on a white background. The target letter was presented at the center of the screen in a lowercase Courier 21-point font and was equally likely to be either an X or a Z. The distractor letter was presented in an uppercase Courier 22-point font either $2 \mathrm{~cm}$ above or $2 \mathrm{~cm}$ below the target (with equal likelihood in each position) and was equally likely to be either an $\mathrm{X}$ or a $\mathrm{Z}$ for each of the $\mathrm{X}$ or $\mathrm{Z}$ targets (to produce $50 \%$ compatible, $50 \%$ incompatible distractor conditions).

\section{Procedure}

Two-task condition. Each trial began with the word "ready" presented in the middle of the screen for $500 \mathrm{msec}$. This was followed by a blank screen and a sequence of five sounds, with a 200-msec interval between each tone. Immediately after the final tone, a question mark was presented until response. The subjects were asked to use their left hand for the auditory task response and to press the "S" or "D" key on the computer keyboard (using adjacent fingers) to indicate whether the low-frequency target tone was long or short. If no response was made within a 2-sec duration from the onset of the last tone, or if an error was made, a beep sound was given by way of feedback.

Following response (or the error feedback), a fixation dot was displayed at the center of the display for $500 \mathrm{msec}$. The flanker task 
display was then presented for $200 \mathrm{msec}$, followed by an 1,800-msec blank interval. The subjects were asked to use their right hand for the flanker task response and to use the numerical keypad to press the " 0 " key with their thumb for a target letter X and the " 2 " key with their middle finger for a target letter $Z$. Feedback for errors or missed responses was again given with a beep sound. The subjects' responses (or feedback, in the case of a missed response) triggered an intertrial interval of $1,200 \mathrm{msec}$.

Single-task condition. The single task condition was the same as the two-task condition, except for instructing the subjects to ignore the sounds, replacing the word "ready" with an asterisk at the start of each trial and replacing the question mark following the sounds with a blank interval that timed out following $2 \mathrm{sec}$ for the onset of the last sound.

Each of the two-task and single-task blocks consisted of a random mix of 72 trials. The experiment started with two short 10 -trial blocks of the single- and two-task conditions, followed by six experimental blocks (three for each condition). Blocks of either the two-task or the single-task condition alternated. Half of the subjects started with a single-task block, and the other half with a two-task block.

The subjects were instructed to fixate on the display center (marked by the central fixation dot before the visual task and the central word "ready" before the auditory task) throughout each trial. They were also requested to try their best to ignore the irrelevant distractor in the flanker task and to make their target response as quickly and accurately as possible. An arbitrary response mapping was used to discourage the subjects from moving their eyes from the screen to the keyboard (as could have been the case had we mapped the task letters to the corresponding keyboard letters). The subjects were reminded of the response mapping in each of the tasks before they embarked on each block of trials, both during the practice and during the experiment, and the subjects were instructed to keep their fingers on these keys throughout block performance.

\section{Results and Discussion}

The subjects who made more than $30 \%$ errors in the auditory discrimination task were excluded from the data in all the experiments reported. This resulted in excluding 4 subjects from this experiment (for whom the mean percentages of errors [PEs] in the auditory discrimination task were $31 \%$, $37 \%, 43 \%$, and $51 \%$ ). For the remaining subjects, the mean reaction time (RT) for the correct responses in the auditory task was 406 msec, and the mean error rate was 17.7\%.

\section{Flanker Task}

Table 1 presents the mean RTs and PEs in the flanker task. Trials with incorrect responses in either the flanker task or the auditory task were removed from the RT analyses. Withinsubjects ANOVAs with the factors of task condition (single task or two tasks) and distractor compatibility (compatible or incompatible) were conducted on the mean RTs and PEs.

The RT ANOVA revealed main effects of distractor compatibility $\left[F(1,13)=48.7, M S_{\mathrm{e}}=1,475, p<.001\right]$ and task condition $\left[F(1,13)=7.4, M S_{\mathrm{e}}=3,779, p<\right.$ $.05]$. RTs were longer in the incompatible distractor condition $(M=614 \mathrm{msec})$ than in the compatible distractor condition $(M=542 \mathrm{msec})$ and in the two-task condition $(M=600 \mathrm{msec})$ than in the single-task condition $(M=$ $555 \mathrm{msec}$ ). Importantly, there was a significant interaction between distractor compatibility and task condition $\left[F(1,13)=6.9, M S_{\mathrm{e}}=840, p<.05\right]$. Distractor compatibility effects were larger in the two-task condition than in the single-task condition (see Table 1), as we predicted.

The increase in overall RTs in the two-task condition is unlikely to account for the greater distractor interference in
Table 1

Mean Reaction Times (RTs, in Milliseconds) and Percentages of Errors (PEs) in the Flanker Task of Experiment 1 As a Function of Task Condition and Distractor Compatibility

\begin{tabular}{|c|c|c|c|c|c|c|}
\hline \multirow[b]{3}{*}{ Task Condition } & \multicolumn{6}{|c|}{ Distractor Compatibility } \\
\hline & \multicolumn{2}{|c|}{ I } & \multicolumn{2}{|c|}{$\mathrm{C}$} & \multicolumn{2}{|c|}{$\mathrm{I}-\mathrm{C}$} \\
\hline & RT & $\mathrm{PE}$ & $\mathrm{RT}$ & $\mathrm{PE}$ & RT & $\mathrm{PE}$ \\
\hline Two tasks & 646 & 11.9 & 554 & 6.7 & 92 & 5.2 \\
\hline Single task & 581 & 6.8 & 529 & 4.8 & 52 & 2.0 \\
\hline
\end{tabular}

Note-I, incompatible; C, compatible.

this condition, because the distractor interference was still greater in the two-task condition than in the single-task condition when calculated as a percentage of the overall RT per individual in each of the task conditions. The mean distractor effect was $9.5 \%$ of the overall RT in the single-task condition and $15.4 \%$ of the overall RT in the two-task condition $[t(13)=2.4, p<.05$, one-tailed, for the difference $]$.

Effects on error rates mirrored those on RTs. The mean $\mathrm{PE}$ was larger in the incompatible distractor condition $(10 \%)$ than in the compatible distractor condition $(6 \%)$ $\left[F(1,13)=9.7, M S_{\mathrm{e}}=18, p<.05\right]$ and was larger in the two-task condition $(10 \%)$ than in the single-task condition $(6 \%)\left[F(1,13)=11.5, M S_{\mathrm{e}}=15, p<.005\right]$. The interaction between both factors was not significant $\left[F(1,13)=3.0, M S_{\mathrm{e}}=12, p=.11\right]$, but the numerical trends were for a larger distractor effect on errors in the two-task condition ( $\mathrm{I}-\mathrm{C}=5 \%$ ) than in the single-task condition ( $\mathrm{I}-\mathrm{C}=2 \%)$, as in the RTs.

\section{EXPERIMENT 2}

Experiment 1 established that task coordination across different modalities leads to increased distraction, in support of predictions derived from the load theory of attention. It remains possible, however, that the effects of task coordination on distraction are larger when both of the tasks that require coordination are within the same modality. Task stimuli within the same modality are more similar to each other than are task stimuli in different modalities, and this may lead to a greater demand on executive control when different stimulus-processing rules have to be coordinated for task stimuli that are more similar to each other. In Experiment 2, we therefore compared the effects of single- versus two-task conditions on distraction levels between a within-modality two-task condition (in which both the tasks were visual) and a between-modality (auditory and visual) two-task condition.

The subjects were simultaneously presented with auditory stimuli (the same as those used in Experiment 1) and visual stimuli (a sequence of hash symbols of varying color and size) prior to the visual flanker task on each trial. Half of the subjects performed the tasks in the between-modality twotask condition (performed the auditory discrimination task, while ignoring the visual discrimination task stimuli, prior to the flanker task on each trial, as in Experiment 1), and the other half performed the tasks in the within-modality twotask condition (performed a visual discrimination task, while ignoring the auditory-discrimination task stimuli, prior to the flanker task on each trial). In the single-task condition, all 
the subjects just performed the flanker task and ignored both the visual and the auditory discrimination task stimuli.

\section{Method}

\section{Subjects}

Forty new paid volunteers ( 20 in each of the two-task modality conditions) from the same pool as that in Experiment 1 participated.

\section{Stimuli and Procedure \\ The flanker task and the sound stimuli used were the same as those in Experiment 1. At the onset of each sound, a visual hash symbol ("\#") was presented for $100 \mathrm{msec}$ in Century font with a size of either 66 or 30 pixels. The target was in gray, and the non- target symbols in black, presented against a white background. In the within-modality two-task condition, the subjects were asked to use their left hand and press " $F$ " or " $G$ " with adjacent fingers on the computer keyboard to indicate whether the gray target symbol was big or small. The auditory task in the cross-modal two-task condition and the single-task condition for all the subjects was the same as that in Experiment 1. In each condition, the subjects were instructed to ignore the irrelevant discrimination task stimuli (i.e., both of the vi- sual and auditory stimuli in the single-task condition or just stimuli in the irrelevant modality in the two-task conditions). \\ All the subjects performed in the single-task condition. Half of the subjects also performed in the between-modality two-task condition, and the other half also performed in the within-modality two-task condition. The subjects were allocated randomly to the two-task mo- dality conditions. As in Experiment 1, six experimental blocks of 72 trials preceded by two 10 -trial practice blocks were performed, with the single-task and two-task conditions presented in alternating block order. Half the subjects started with a single-task block, and the other half with a two-task block.}

\section{Results and Discussion}

Three subjects with a mean error rate of $37 \%$ each and 1 with a mean error rate of $48 \%$ in the auditory discrimination task were excluded from further analyses. One-way between-subjects ANOVAs on the RTs and PEs in the auditory and visual discrimination tasks revealed no difference $(F<1)$ in the mean RTs (mean RT was 465 and $478 \mathrm{msec}$ for the visual task and the auditory task, respectively), but the mean percentage of errors was higher in the auditory task (mean $\mathrm{PE}=13.8 \%$ ) than in the visual task (mean $\mathrm{PE}=4.9 \%)\left[F(1,34)=12.9, M S_{\mathrm{e}}=54, p<.001\right]$.

\section{Flanker Task}

Table 2 presents the mean RTs and error rates in the flanker task. Trials with incorrect responses in either the flanker task or the discrimination task were removed from the RT analyses. Mixed model ANOVAs with the within-subjects factors of task condition (single task or two tasks) and distractor compatibility (compatible or incompatible) and the betweensubjects factor of two-task modality (visual or auditory) were conducted on the mean RTs and error rates.

The RT ANOVA revealed main effects of distractor compatibility $\left[F(1,34)=56.9, M S_{\mathrm{e}}=1,844, p<.001\right]$ and task conditions $\left[F(1,34)=12.4, M S_{\mathrm{e}}=4,444, p<.001\right]$. RTs were longer in the incompatible distractor condition $(M=$ $614 \mathrm{msec})$ than in the compatible distractor condition $(M=$ $559 \mathrm{msec})$ and in the two-task condition $(M=606 \mathrm{msec})$ than in the single-task condition $(M=567 \mathrm{msec})$.

Importantly, there was an interaction between distractor compatibility, task condition, and two-task modality
$\left[F(1,34)=5.6, M S_{\mathrm{e}}=698, p<.05\right]$. This interaction reflected the different effects of task condition on distractor compatibility between the within-modality and the between-modality two-task conditions. In the betweenmodality two-task condition, distractor compatibility effects were greater in the two-task than in the single-task condition $\left[F(1,34)=4.6, M S_{\mathrm{e}}=698, p<.05\right]$, as before. This effect was also found when distractor effects were calculated as percentages of the overall RT per individual in the single-task condition $(M=9.1 \%)$ and the two-task condition $(M=13.1 \%)[t(15)=2.5, p<.05$, one-tailed, for the difference]. But in the within-modality two-task condition, distractor compatibility effects were not significantly different in the two- versus single-task conditions $\left[F(1,34)=1.3, M S_{\mathrm{e}}=698, p=.25\right]$.

There were no other significant two-way interactions for the RTs (all $F \mathrm{~s}<2.2, p \mathrm{~s}>.15$ ), and the error rates showed only a distractor compatibility effect $[F(1,34)=$ 11.7, $M S_{\mathrm{e}}=20, p<.005$; all other $\left.F \mathrm{~s}<1\right]$.

In conclusion, somewhat surprisingly, the effects of twotask coordination led to increased distraction only when the two tasks involved different (visual and auditory) modalities, but not when the two tasks involved the same (visual) modality. We note that the failure to find any increase in distraction in the within-modality two-task condition (as compared with the single-task condition) appears to contradict Brand and Lavie's (2005) recent finding that switching between two visual tasks (as compared with repeatedly performing the same task) does lead to greater distractor interference in the response competition paradigm. Although the difference between the tasks used in addition to the flanker task here (a visual discrimination task) and in Brand and Lavie's (2005) study (a visual search task) precludes a direct comparison, it is important to note that in another study, Brand and Lavie (2006) have also established that the effects of task switching on the level of distractor interference are not found when each task is mapped to a different hand, as indeed was the case in the present study.

In addition, the contrast in the results for the effects of executive load on distraction between the within- and the between-modality conditions raises the possibility that task coordination between the different modalities may involve a greater demand on executive control (and hence, may lead to greater effects on distractibility) than does task coordination within the same modality. This possibility is intriguing and has received some support in previous research (e.g., Hunt \& Kingstone, 2004). However, recall that the error rates were significantly higher in the auditory discrimination task than in the visual discrimination task. It is thus possible that the difference between the withinmodality and the between-modality conditions is due to their different level of discrimination task difficulty. This issue was addressed in the following experiments.

\section{EXPERIMENT 3}

In Experiment 3, we examined whether the coordination of two tasks within the same modality would increase distractor interference when the visual discrimination task used was more demanding. Subjects now had to search for 
Table 2

Mean Reaction Times (RTs, in Milliseconds) and Percentages of Errors (PEs) in the Flanker Task of Experiment 2 As a Function of the Two-Task Modality Condition, Task Condition, and Distractor Compatibility

\begin{tabular}{|c|c|c|c|c|c|c|c|c|c|c|c|c|}
\hline \multirow{4}{*}{$\begin{array}{c}\text { Task } \\
\text { Condition }\end{array}$} & \multicolumn{12}{|c|}{ Two-Task Modality Condition } \\
\hline & \multicolumn{6}{|c|}{ Between Modalities } & \multicolumn{6}{|c|}{ Within Modality } \\
\hline & \multicolumn{2}{|c|}{ I } & \multicolumn{2}{|c|}{$\mathrm{C}$} & \multicolumn{2}{|c|}{$\mathrm{I}-\mathrm{C}$} & \multicolumn{2}{|c|}{$\mathrm{I}$} & \multicolumn{2}{|c|}{$\mathrm{C}$} & \multicolumn{2}{|c|}{$\mathrm{I}-\mathrm{C}$} \\
\hline & RT & $\mathrm{PE}$ & RT & $\mathrm{PE}$ & RT & $\mathrm{PE}$ & RT & PE & RT & $\mathrm{PE}$ & RT & $\mathrm{PE}$ \\
\hline Two tasks & 619 & 5.6 & 540 & 3.3 & 79 & 2.3 & 651 & 7.2 & 614 & 4.7 & 37 & 2.5 \\
\hline Single task & 579 & 5.5 & 528 & 3.0 & 51 & 2.5 & 605 & 7.9 & 555 & 4.7 & 50 & 3.2 \\
\hline
\end{tabular}

Note-I, incompatible; C, compatible.

a conjunction target: an upright or inverted $\mathrm{T}$ with a vertical red bar and a horizontal blue bar (among other upright and inverted Ts that had the opposite color-orientation combination) and decide which of the bars was longest, the horizontal or the vertical.

\section{Method}

\section{Subjects}

Thirty psychology students from the Université de Provence received course credit for their participation in this experiment.

\section{Stimuli and Procedure}

The sound and flanker task stimuli were the same as those in Experiment 2, and the procedure was the same as that used in the within-modality single-task and two-task conditions in Experiment 2 . The main changes concerned the visual discrimination task. The \# symbols were replaced with upright or inverted Ts. The target had a vertical red bar and a horizontal blue bar, and the nontarget Ts had the opposite combination. Each of the horizontal and vertical bars in each T was 6 pixels wide and equally likely to be either 35 or 44 pixels long. The subjects were told to ignore the sounds and, in the two-task condition, to press the "S" or " $D$ " key with adjacent fingers to indicate whether the longer target bar was vertical or horizontal. In the single-task condition, they were told to ignore the Ts as well; in all other respects, the single-task procedure was the same as that in Experiments 1 and 2.

\section{Results and Discussion}

Four subjects with mean PEs of $53 \%, 51 \%, 47 \%$, and $45 \%$ in the visual discrimination task were excluded from any further analysis. The mean RT of the correct responses in the auditory task was $484 \mathrm{msec}$, and the mean error rate was $11.7 \%$. These indicate a level of difficulty similar to that of the auditory discrimination task in Experiment 1 (in which the mean RT was $406 \mathrm{msec}$ and the mean PE was $17.7 \%$ ) and Experiment 2 (in which the mean RT was $478 \mathrm{msec}$ and the mean PE was 14\%).

\section{Flanker Task}

Table 3 presents the mean RTs and error rates in the flanker task. Trials with incorrect responses in either the flanker task or the visual discrimination task were removed from the RT analyses. Within-subjects ANOVAs with the factors of task condition (single task or two tasks) and distractor compatibility (compatible or incompatible) were conducted on the mean RTs and error rates.

The RT ANOVA revealed main effects of distractor compatibility $\left[F(1,23)=42.8, M S_{\mathrm{e}}=3,490, p<.001\right]$ and task conditions $\left[F(1,23)=68.9, M S_{\mathrm{e}}=6,570, p<\right.$
.001]: RTs were longer in the incompatible distractor condition $(M=623 \mathrm{msec})$ than in the compatible distractor condition $(M=544 \mathrm{msec})$ and in the two-task condition $(M=652 \mathrm{msec})$ than in the single-task condition $(M=$ $514 \mathrm{msec}$ ). Importantly, there was now a significant interaction between distractor compatibility and task condition $\left[F(1,23)=8.4, M S_{\mathrm{e}}=1,183, p<.01\right]$, so that distractor compatibility effects in this experiment were larger in the two-task condition than in the single-task condition (see Table 3), and this effect was also found when distractor effects were calculated as percentages of the overall RT per individual, in the single-task condition $(M=11.3 \%)$ and in the two-task condition $(M=14.8 \%)[t(23)=2.9, p<.05$, one-tailed, for the difference]. Clearly, the effects of task coordination on susceptibility to distraction can be found when the flanker task is coordinated with another task within the same (visual) modality, even when the tasks use separate hands (cf. Brand \& Lavie, 2006), as long as the additional, coordinated visual task is sufficiently demanding.

Effects on error rates mirrored those on RTs. The mean $\mathrm{PE}$ was larger in the incompatible distractor condition $(M=$ $8 \%)$ than in the compatible distractor condition $(M=4 \%)$ $\left[F(1,23)=14.9, M S_{\mathrm{e}}=26, p<.001\right]$ and in the twotask condition $(M=7 \%)$ than in the single-task condition $(M=4 \%)\left[F(1,23)=8.4, M S_{\mathrm{e}}=27, p<.01\right]$. Distractor compatibility effects tended to be greater in the two-task condition ( $\mathrm{I}-\mathrm{C}=6 \%)$ than in the single-task condition $(\mathrm{I}-\mathrm{C}=3 \%)\left[F(1,23)=3.1, M S_{\mathrm{e}}=16, p=.08\right]$.

\section{EXPERIMENT 4}

Although task difficulty clearly affects the extent to which task coordination within the same modality loads on executive control, this may not be the case for task coordination across different modalities. Indeed, Experiment 2 hinted at the possibility that task coordination be-

Table 3

Mean Reaction Times (RTs, in Milliseconds) and Percentages of Errors (PEs) in the Flanker Task of Experiment 3 As a Function of Task Condition and Distractor Compatibility

\begin{tabular}{|c|c|c|c|c|c|c|}
\hline \multirow[b]{3}{*}{ Task Condition } & \multicolumn{6}{|c|}{ Distractor Compatibility } \\
\hline & \multicolumn{2}{|c|}{ I } & \multicolumn{2}{|c|}{$\mathrm{C}$} & \multicolumn{2}{|c|}{$\mathrm{I}-\mathrm{C}$} \\
\hline & RT & $\mathrm{PE}$ & RT & PE & RT & $\mathrm{PE}$ \\
\hline Two tasks & 701 & 10.0 & 602 & 4.5 & 99 & 5.5 \\
\hline Single task & 544 & 5.4 & 485 & 2.8 & 59 & 2.6 \\
\hline
\end{tabular}

Note-I, incompatible; C, compatible. 
tween (as compared with within) different modalities may involve a greater demand on executive control, so that its effects on the level of distraction may be insensitive to task difficulty. In Experiment 4, we tested this hypothesis by requesting subjects to coordinate the visual flanker task with an easy auditory detection task.

As in Experiment 1, the subjects performed the flanker task either in a single-task condition or in a two-task condition in which the flanker task immediately followed an auditory task. But in a change from Experiment 1, the auditory task was far easier, requiring the subjects to detect the presence or absence of an odd low-pitch $(440-\mathrm{Hz})$ target among four higher pitch $(520-\mathrm{Hz})$ sounds. If task coordination between modalities is more demanding on executive control, as compared with task coordination within the same modality, greater distractor interference effects in the two-task condition (as compared with the singletask condition) should be found here, despite the use of an easy auditory task with a level of difficulty comparable to that of the easy visual task in Experiment 2.

\section{Method}

\section{Subjects}

Twenty-two new subjects from the Université de Provence received course credit for their participation in this experiment.

\section{Stimuli and Procedure}

The stimuli and procedure were the same as those in Experiment 1, except for the following changes. The target tone was always a $440-\mathrm{Hz}$ tone presented for a duration of $150 \mathrm{msec}$. The target was present on $50 \%$ of the trials (in any sequence position [except the first], selected at random) and was absent on the other $50 \%$ of the trials. The present and absent trials were intermixed in random order within a block. In the two-task condition, the subjects were requested to press the "S" key (with the middle finger of their left hand) to indicate that the lowfrequency target tone was present and the "D" key (with the index finger of their left hand) to indicate that it was absent.

\section{Results and Discussion}

\section{Auditory Discrimination Task}

Two subjects with mean PEs of $37 \%$ and $31 \%$ in the auditory discrimination task were excluded from any further analyses. For the remaining subjects, the mean RT in the auditory discrimination task was $388 \mathrm{msec}$, and the mean error rate was $5.6 \%$. These results suggest that the auditory discrimination task used in this experiment was easier than any of the visual or auditory discrimination tasks used in the previous experiments. Specifically, with a mean RT $(388 \mathrm{msec})$ that was similar to (although numerically shorter than) that of the auditory discrimination task in Experiment $1(M=406 \mathrm{msec})$, the mean error rate $(5.6 \%)$ was significantly smaller $[t(32)=4.4, p<$ $.001]$ than that in Experiment 1 (for which the mean PE was $17.7 \%$ ); and with a mean error rate similar to that in the easy visual discrimination task used in Experiment 2 $(M=4.9 \%)$, the mean RT was significantly shorter (mean RT for the easy visual discrimination task in Experiment 2 was $465 \mathrm{msec}$ ) $[t(38)=2.4, p<.05$, for the difference].

\section{Flanker Task}

Table 4 presents the mean RTs and PEs in the flanker task. Trials with incorrect responses in either the flanker task or the auditory discrimination task were removed from the RT analyses. Within-subjects ANOVAs with the factors of task condition (single task or two tasks) and distractor compatibility (compatible or incompatible) were conducted on the mean RTs and PEs.

The RT ANOVA revealed main effects of distractor compatibility $\left[F(1,19)=13.3, M S_{\mathrm{e}}=3,989, p<.001\right]$ and task condition $\left[F(1,19)=6.81, M S_{\mathrm{e}}=6,412, p<\right.$ $.05]$ : RTs were longer in the incompatible distractor condition $(M=500 \mathrm{msec})$ than in the compatible distractor condition $(M=448 \mathrm{msec})$ and in the two-task condition $(M=497 \mathrm{msec})$ than in the single-task condition $(M=$ $451 \mathrm{msec}$ ). Importantly, there was also a significant interaction between distractor compatibility and task condition $\left[F(1,19)=5.8, M S_{\mathrm{e}}=551, p<.05\right]$, so that distractor compatibility effects in this experiment were larger in the two-task condition than in the single-task condition (see Table 4) and this effect was also found when distractor effects were calculated as percentages of the overall RT per individual, in the single-task condition $(M=6.6 \%)$ and in the two-task condition $(M=11.3 \%)[t(19)=2.3, p<$ .05 , one-tailed, for the difference].

Clearly, even with the addition of an easy auditory task (of a difficulty comparable to that of the easy visual task in Experiment 2), the effects of two-task coordination on susceptibility to distraction can be found. Moreover, the increase in the distractor interference effects in the twotask condition in this experiment was, in fact, greater than that found in the within-modality two-task condition with a difficult visual discrimination task in Experiment 3. The overall mean RT in the flanker task in this experiment was shorter than that in Experiment 3, but an inspection of the percentage of distractor compatibility effects in each experiment showed that the magnitude of the increase in percentage of distractor effects in the two-task condition was $71 \%$ of the percentage of distractor effects in the single-task condition (an increase from $6.6 \%$ to $11.3 \%$ ) in Experiment 4 and only a $33 \%$ increase in percentage of distractor effects in the singletask condition (an increase from $11.3 \%$ in the single-task condition to a mean of $14.8 \%$ in the two-task conditions) in Experiment 3.

Mean error rate was larger in the incompatible distractor condition $(5 \%)$ than in the compatible distractor condition $(2 \%)[F(1,19)=5.6, p<.05]$ and was larger in the two-task condition $(4.6 \%)$ than in the single-task condition $(3.2 \%)[F(1,19)=6.3, p<.05]$. The interaction between both factors was not significant $(F<1)$.

\section{Table 4}

Mean Reaction Times (RTs, in Milliseconds) and Percentages of Errors (PEs) in the Flanker Task of Experiment 4 As a Function of Task Condition and Distractor Compatibility

\begin{tabular}{|c|c|c|c|c|c|c|}
\hline \multirow[b]{3}{*}{ Task Condition } & \multicolumn{6}{|c|}{ Distractor Compatibility } \\
\hline & \multicolumn{2}{|c|}{$\mathrm{I}$} & \multicolumn{2}{|c|}{$\mathrm{C}$} & \multicolumn{2}{|c|}{$\mathrm{I}-\mathrm{C}$} \\
\hline & RT & $\mathrm{PE}$ & RT & $\mathrm{PE}$ & RT & $\mathrm{PE}$ \\
\hline Two tasks & 529 & 5.7 & 465 & 3.6 & 64 & 2.1 \\
\hline Single task & 470 & 4.3 & 431 & 2.1 & 39 & 2.2 \\
\hline
\end{tabular}

Note-I, incompatible; C, compatible. 


\section{GENERAL DISCUSSION}

The present experiments extend load theory to account for distractibility during two-task coordination across different sensory modalities (vision and hearing), as well as within the visual modality. The results provide support for the load theory prediction that executive control load leads to greater distractor interference while highlighting the effects of task modalities, as well as the level of task difficulty.

\section{Task Coordination and the Level of Distraction: Support for Load Theory}

In all but one (the easy visual discrimination task, Experiment 2) of our tasks, the two-task (as compared with single-task) conditions resulted in increased response competition effects by the irrelevant distractor in the flanker task. These findings provide support for our load theory prediction that the demand placed on executive control by the need to coordinate the two-task alternation would reduce the availability of executive control to oversee task performance so that it is goal directed, with minimal distraction by irrelevant distractors.

Although the findings provide direct support for this prediction and the coordination of task alternation, or task switching, is known to draw on executive control (e.g., Rubinstein, Meyer, \& Evans, 2001; see Monsell, 2003, for a review), it is worth considering whether it is the demand on executive control per se that led to the increased distraction in our tasks or whether the increased distractor effects were due to other aspects of the task. For example, the two-task condition involved alternation from one response set to another (whereas there was only one response set in the singletask condition), and despite the 500-msec fixation display prior to the flanker task, it remains possible that the flanker task set was not fully instigated in the two-task condition. One might, therefore, wonder whether the greater distractor interference effect in the flanker task is somehow due to the task set and response mappings not being fully instigated in the two-task condition, rather than to the increased demands these processes impose on executive control.

However, alternative accounts in such terms seem unlikely, given that distractor interference was measured here through response competition effects and these depend on the strength and speed of activation of the assigned distractor response. A weaker response mapping in the two-task condition or an unready response due to the task set's not being fully instigated cannot explain a greater or faster activation of the distractor response that is implied by the greater magnitude of response competition effects in the two-task condition.

Another aspect of the task set preparation concerns the distinction between the target and the distractor letters. In our flanker task, this was based on their locations (the target location was central, whereas the distractor location was always more peripheral). The discrimination tasks preceding the flanker task involved central presentation of the task stimuli (either a rapid serial visual presentation at fixation in the visual discrimination task or tones perceived as coming from the center of the head, with a balanced left- and right-headphone presentation, in the auditory discrimination task) and were always followed by the 500-msec fixation display. It is therefore unlikely that the discrimination task performance would have directly affected the locational certainty between target and distractors or the subjects' ability to use the fixation display and attend to the central targets in the flanker task.

It is, therefore, the increased demand on executive control that task coordination involves that appears more likely to account for the present results, instead of some other specific aspects of task alternation in our design. An account in terms of the role of executive control in determining distractor processing can also accommodate a wider range of evidence that has been obtained in other tasks involving working memory load and showing activation of the prefrontal cortices associated with such executive control functions, as was discussed in the introduction.

\section{Effects of Task Modality and Level of Difficulty}

The results highlighted an important difference between task coordination across the visual and auditory modalities and within the visual modality only. Greater interference effects by irrelevant distractors were found in the visual flanker task when it was coordinated with the auditory tone discrimination task, regardless of the level of task difficulty in the auditory discrimination task.

By contrast, for two-task coordination within the visual modality, the effects on the level of distraction were found to critically depend on the difficulty of the coordinated task. Distractor interference was increased only with the coordination of a more difficult visual discrimination task (Experiment 3) but remained unaffected with the coordination of an easier visual task (Experiment 2).

These results suggest that task coordination involving different sensory modalities is more taxing on executive control and, hence, more prone to distraction than is task coordination within the same modality. Although the suggestion that task coordination across different (as compared with the same) sensory modalities is more taxing on executive control concurs with similar suggestions in previous research (e.g., Hunt \& Kingstone, 2004), it may appear somewhat surprising in the light of previous findings that attention task performance is, in fact, more efficient for two sources of input in different (vs. the same) modalities. For example, Treisman and Davies (1973) found that searching for a target among nontargets in a different modality was more efficient than searching for the same target among nontargets in the same modality. Duncan, Martens, and Ward (1997) found that attentional blink - namely, interference in identification of a second target in a stream - was found when subjects attended to two streams of letters in the same modality (e.g., two visual streams or two auditory streams), but not when the two streams were in different modalities (i.e., a visual stream combined with an auditory stream).

The apparent discrepancy between our findings and these previous findings can be resolved by pointing to the difference between the demands on divided attention abilities (e.g., sharing perceptual capacity) made by concurrent sources of input in the previous studies and the demands on the higher level executive control function involved in the coordination of alternation between two 
successive tasks, each with a different input as well as different task rules, in the present study.

Some of the divided attention resources, such as perceptual capacity, may be modality specific and, therefore, can be more efficiently shared between concurrent inputs in the different modalities. Such an account can also accommodate the previous findings that high auditory load has no effect on the processing and brain activity related to visual motion distractors (Rees, Frith, \& Lavie, 2001); these were reduced only with high visual load.

In contrast to the efficiency of dividing perceptual capacity between concurrent inputs in the different modalities, the present study has shown the reduced efficiency of higher level executive control of task coordination when this involves different sensory modalities. Clearly, although executive control functions are in themselves higher level amodal processes, the requirement to coordinate alternation of input between the different modalities does impose extra executive load, thus resulting in a greater vulnerability to distraction.

These findings are important in extending load theory to account for distractibility during task coordination within and between sensory modalities and also have some important implications for the design of applied settings: Reduced distraction in situations that involve multipletask coordination can be obtained by attempting to keep successive tasks within the same sensory modality.

Finally, it is also important to note that the two tasks we have used shared no content, either when both of them were in the same modality or when they involved different sensory modalities (letters were presented in the flanker task, whereas colored hash symbols and tones were presented in the visual or auditory discrimination task, respectively). Thus, the effects of executive control on distractibility established here cannot be due to any content-specific effects of load (cf. Kim, Kim, \& Chun, 2005). Instead, the present findings support a general role for executive control in determining irrelevant distraction that extends to tasks in the different modalities.

Fruitful avenues for future research would involve clarifying further the effects of executive control on distraction, addressing issues such as the specific level of distractor processing that is increased by executive load (e.g., whether executive load increases perceptual processing of the distractors or just their effects of response selection), as well as how executive load and perceptual load interact in the case of between-modality tasks (see Lavie et al., 2004, for their interaction in within-modality tasks). The generality of the effects of executive load on distraction should also be examined by testing whether the effects can replicate across other modalities and modality combinations (e.g., combining tactile tasks with visual or auditory tasks).

\section{AUTHOR NOTE}

This research was supported by an Individual European Community Marie Curie Fellowship to the first author (HPMF-CT-2002-02092), held in the lab of the second author, and by a project grant from the Wellcome Trust to the second author. Correspondence concerning this article should be addressed to N. Lavie, Department of Psychology, Uni- versity College London, Gower Street, London WC1E 6BT, England (e-mail: n.lavie@ucl.ac.uk).

\section{REFERENCES}

BAdDEley, A. D. (1996). Exploring the central executive. Quarterly Journal of Experimental Psychology, 49A, 1-28.

BRAND, S., \& LAVIE, N. (2005, January). Distractor interference during task switching. Paper presented to the Experimental Psychological Society, London.

Brand, S., \& Lavie, N. (2006, January). Task switching, distractor interference and stimulus-response mappings. Paper presented to the Experimental Psychological Society, London.

de Fockert, J. W., Rees, G., Frith, C. D., \& Lavie, N. (2001). The role of working memory in visual selective attention. Science, 291, 1803-1806.

Desimone, R., \& Duncan, J. (1995). Neural mechanisms of selective visual attention. Annual Review of Neuroscience, 18, 193-222.

DunCAN, J., Martens, S., \& WARD, R. (1997). Restricted attentional capacity within but not between sensory modalities. Nature, 387, 808-810.

Eriksen, B. A., \& ERIKSEN, C. W. (1974). Effects of noise letters upon the identification of a target letter in a nonsearch task. Perception \& Psychophysics, 16, 143-149.

HAN, S. H., \& KIM, M. S. (2004). Visual search does not remain efficient when executive working memory is working. Psychological Science, 15, 623-628.

Hunt, A. R., \& Kingstone, A. (2004). Multisensory executive functioning. Brain \& Cognition, 55, 325-327.

Kiм, S.-Y., Kiм, M.-S., \& Chun, M. M. (2005). Concurrent working memory load can reduce distraction. Proceedings of the National Academy of Sciences, 102, 16524-16529.

Lavie, N. (1995). Perceptual load as a necessary condition for selective attention. Journal of Experimental Psychology: Human Perception \& Performance, 21, 451-468.

LAVIE, N. (2000). Selective attention and cognitive control: Dissociating attentional functions through different types of load. In S. Monsell \& J. Driver (Eds.), Attention and performance XVIII: Control of cognitive processes (pp. 175-194). Cambridge, MA: MIT Press.

Lavie, N. (2005). Distracted and confused? Selective attention under load. Trends in Cognitive Sciences, 9, 75-82.

LAVIe, N., \& DE FockerT, J. W. (2005). The role of working memory in attentional capture. Psychonomic Bulletin \& Review, 12, 669-674.

LAVIE, N., \& DE FockerT, J. [W.] (2006). Frontal control of attentional capture in visual search. Visual Cognition, 14, 863-876.

Lavie, N., Hirst, A., De Fockert, J. [W.], \& Viding, E. (2004). Load theory of selective attention and cognitive control. Journal of Experimental Psychology: General, 133, 339-354.

Logan, G. D. (1978). Attention in character-classification tasks: Evidence for the automaticity of component stages. Journal of Experimental Psychology: General, 107, 32-63.

Monsell, S. (2003). Task switching. Trends in Cognitive Sciences, 7, 134-140.

Rees, G., Frith, C. D., \& Lavie, N. (2001). Processing of irrelevant visual motion during performance of an auditory task. Neuropsychologia, 39, 937-949.

Rubinstein, J. S., Meyer, D. E., \& Evans, J. E. (2001). Executive control of cognitive processes in task switching. Journal of Experimental Psychology: Human Perception \& Performance, 27, 763-797.

Treisman, A. M., \& Davies, A. (1973). Divided attention to ear and eye. In S. Kornblum (Ed.), Attention and performance IV (pp. 101-117). New York: Academic Press.

Woodman, G. F., \& Luck, S. J. (2004). Visual search is slowed when visuospatial working memory is occupied. Psychonomic Bulletin \& Review, 11, 269-274.

Woodman, G. F., Vogel, E. K., \& Luck, S. J. (2001). Visual search remains efficient when visual working memory is full. Psychological Science, 12, 219-224.

(Manuscript received July 5, 2006; revision accepted for publication November 5, 2007.) 